\title{
Protective role of tenuigenin on sepsis-induced acute kidney injury in mice
}

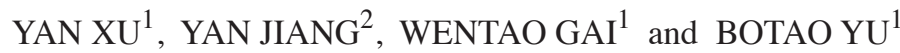 \\ Departments of ${ }^{1}$ Urinary Surgery and ${ }^{2}$ Burns and Plastic Surgery, \\ Laiyang Central Hospital of Yantai, Yantai, Shandong 265200, P.R. China
}

Received March 29, 2016; Accepted March 3, 2017

DOI: $10.3892 /$ etm.2017.5164

\begin{abstract}
Acute kidney injury (AKI) is a severe complication of sepsis, which largely contributes to the associated high mortality rate. Tenuigenin (TNG) is a natural product isolated from Polygala tenuifolia root, which possesses anti-inflammatory and anti-oxidant properties. The present study investigated the effects of TNG on sepsis-associated AKI in mice subjected to cecal ligation and puncture (CLP). TNG was demonstrated to alleviate sepsis-induced AKI by reducing pathological changes and significantly decreasing the levels of blood urea nitrogen, serum creatinine and kidney coefficient. The production of inflammatory cytokines, including tumor necrosis factor $\alpha$ and interleukin-6, was markedly inhibited by TNG. Hematoxylin-eosin staining revealed that the morphological changes of kidney tissues in CLP mice were reversed following TNG treatment. Furthermore, treatment with TNG inhibited the production of nitric oxide and prostaglandin $\mathrm{E}_{2}$. Finally, TNG inhibited the activation of the nuclear factor- $\kappa \mathrm{B}$ $(\mathrm{NF}-\kappa \mathrm{B})$ signaling pathway. The present study suggested that TNG alleviates sepsis-induced AKI by inhibiting the NF- $\mathrm{BB}$ signaling pathway, which provides a novel approach for treating sepsis-induced AKI.
\end{abstract}

\section{Introduction}

Acute kidney injury (AKI) is associated with high mortality and occurs in up to $35 \%$ of hospitalized patients (1). In patients who receive general surgery, the incidence of AKI has been reported to be $\sim 1 \%$, while it may occur in up to $70 \%$ of critically ill patients, and if AKI is part of the multiple organ dysfunction syndrome, the in-hospital mortality is $50 \%(2,3)$. AKI is an independent risk factor for mortality (4), and surviving patients have an increased risk of developing chronic kidney

Correspondence to: Mrs. Yan Jiang, Department of Burns and Plastic Surgery, Laiyang Central Hospital of Yantai, 111 Changshan Road, Yantai, Shandong 265200, P.R. China

E-mail: jiangyan0790@126.com

Key words: acute kidney injury, tenuigenin, inflammatory, nitric oxide, nuclear factor- $\kappa \mathrm{B}$ disease. AKI comprises multiple clinical conditions and outcomes are influenced by underlying disease. In critically ill patients, sepsis is the most common cause of AKI. Sepsis is a severe systemic inflammatory response triggered by a bacterial, viral or fungal infection and represents a major cause of shock or mortality (5). Sepsis-induced AKI is characterized by a rapid and often profound decline in the kidney's function to filter blood and eliminate nitrogen waste products, usually evolving over hours to days after the onset of sepsis (6).

At present, no therapeutic measures are available to prevent or treat sepsis-induced AKI due to the limited understanding of the pathophysiological mechanisms. The involvement of inflammation, microvascular dysfunction and adaptive responses of tubular cells in the development of sepsis-induced AKI provides novel therapeutic and diagnostic avenues. Among the numerous pathophysiological processes underlying sepsis, inflammation has prominent roles (7), which results from the activation of nuclear factor- $\kappa \mathrm{B}(\mathrm{NF}-\kappa \mathrm{B})$ through intracellular signaling pathways (8). NF- $\kappa \mathrm{B}$ has been demonstrated to mediate the transcription of a large number of genes, the products of which are known to have important roles in septic pathophysiology (9). Mice with deficiency of these $\mathrm{NF}-\kappa \mathrm{B}$-dependent genes are resistant to the development of septic shock and sepsis-associated mortality (10). Pro-inflammatory cytokines, including interleukin-6 (IL-6) and tumor necrosis factor $\alpha$ (TNF- $\alpha$ ) have been demonstrated to be significantly higher in patients with sepsis than in those without (7). Numerous clinical trials have been performed to modulate the excessive inflammatory response in sepsis-induced AKI cases, but to date, no immunomodulatory drug has been demonstrated to decrease the mortality of patients with severe sepsis-induced AKI (11). Therefore, there is a compelling need to develop novel therapeutic options for patients with sepsis-induced AKI.

Tenuigenin (TNG) is a major active component of the Chinese herb Polygala tenuifolia root, also known as 'Yuan Zhi' according to the Chinese Materia Medica, and is used to treat patients with insomnia, neurosis and dementia (12). TNG has been reported to have various biological and pharmacological activities, including anti-oxidant, anti-inflammatory, anti-dementia and anti-aging effects $(13,14)$. Given that TNG, which is extensively applied in Traditional Chinese Medicine, inhibits the inflammatory response, which has crucial roles in the pathophysiological development of sepsis-induced AKI, 
the present study sought to investigate the ability of TNG to reduce it and assessed whether the underlying mechanism involves the NF-kB pathway.

\section{Materials and methods}

Animals. Adult male Kunming (KM) mice, weighing 25-30 g and 6 weeks old, were obtained from the Experimental Animal Center of Shandong Luye Pharmaceutical Co., Ltd. (Yantai, China). The animals were kept under a 12-h light/dark cycle at a temperature of $22 \pm 2^{\circ} \mathrm{C}$ and $55 \pm 5 \%$ relative humidity. All animal experiments were performed according to the protocols approved by the Animal Care and Use Ethics Committee of Laiyang Central Hospital (Yantai, China) and were in strict accordance with US National Institutes of Health Guide for the Care and Use of Laboratory Animals (15).

Experimental protocol. A total of $100 \mathrm{KM}$ mice were randomly divided into five groups: A Sham group, a cecal ligation and puncture (CLP) model group, a high-dose TNG (H) group, a moderate-dose TNG (M) group and a low-dose TNG (L) group. Mice underwent CLP to mimic sepsis-induced acute kidney injury. Immediately following the sham surgery or CLP, the mice in three TNG treatment groups received TNG (National Institute for the Control of Pharmaceutical and Biological Products, Jilin, China) at a single dose of 50,20 or $5 \mathrm{mg} / \mathrm{kg}$, respectively, by intraperitoneal injection. At $24 \mathrm{~h}$ following CLP or sham surgery, the body weight of all mice was determined. The blood was collected in heparinized centrifuge tubes through the abdominal aorta and following collection of blood samples, a high dose of anesthetic $(110 \mathrm{mg} / \mathrm{kg}$ thiopental sodium, i.p.) was administered and the animals were bled to death. Freshly isolated serum was used for the assessment of renal function. The two kidneys were harvested and weighed, and were used for histological sectioning and biochemical assays.

CLP procedure. CLP was performed according to the procedure used by Rittirsch et al (16). In brief, following anesthetizing mice by intraperitoneal injection $300 \mathrm{mg} / \mathrm{kg}$ chloral hydrate, a midline incision of $2-3 \mathrm{~cm}$ in length was made to expose the cecum and the adjoining intestine. Subsequently, $50 \%$ of the cecum was ligated and perforated between the ligation and the tip of the cecum in a mesenteric to anti-mesenteric direction with an 18-gauge needle. Following extrusion of a small amount of stool through the puncture site, the cecum was replaced into the abdomen and the incision was closed using a sterile 4-0 silk suture. Finally, $30 \mathrm{ml} / \mathrm{kg}$ of warm sterile saline was subcutaneously administered for fluid resuscitation. Mice in the sham group received the same procedure without CLP.

Assessment of renal function. Blood samples were collected from peritoneal veins and centrifuged at $2,500 \mathrm{x} \mathrm{g}$ at $4^{\circ} \mathrm{C}$ for $10 \mathrm{~min}$. The levels of blood urea nitrogen (BUN) and serum creatinine ( $\mathrm{SCr}$ ) were assayed with an automated biochemical analyzer (TBA-40FR; Toshiba, Tokyo, Japan).

Biochemical assays. Each kidney tissue sample was weighed using an analytical balance and $100 \mathrm{mg}$ tissue of each sample was homogenized in $0.01 \mathrm{M}$ PBS buffer ( $\mathrm{pH}$ 7.2). Following centrifugation of the homogenate at $12,000 \mathrm{x}$ g for $30 \mathrm{~min}$ at $4^{\circ} \mathrm{C}$, the supernatant was collected and quantitatively assayed for TNF- $\alpha$ and IL- 6 using tumor necrosis factor- $\alpha$ (cat. no. H052) and interleukin-6 (cat. no. H007) ELISA kits (Nanjing Jiancheng Biological Engineering Institute, Nanjing, China) according to the manufacturer's protocol.

Determination of nitric oxide $(N O)$ and prostaglandin $E_{2}$ $\left(P G E_{2}\right)$. The amount of nitrite (a stable metabolite of $\mathrm{NO}$ ) in the kidney tissues was detected by the Griess Reagent System (Beyotime Institute of Biotechnology, Inc., Haimen, China) according to the manufacturer's protocol. The level of $\mathrm{PGE}_{2}$ in the kidney tissues was detected using a PGE2 ELISA kit (cat. no. KB3797; Shanghai Ke Min Biotechnology Co., Ltd, Shanghai, China, according to the manufacturer's protocol.

Western blot analysis. The kidney samples were homogenised, lysed in radioimmunoprecipitation assay buffer $[50 \mathrm{mmol} / \mathrm{l}$ Tris (pH 7.4), $150 \mathrm{mmol} / \mathrm{l} \mathrm{NaCl}, 1 \%$ Triton $\mathrm{X}-100,1 \%$ sodium deoxycholate, $0.1 \%$ SDS, sodium orthovanadate, sodium fluoride, EDTA and leupeptin] with protease and phosphatase inhibitors, and centrifuged. The resulting supernatants containing cytoplasmic proteins were assayed using a bicinchoninic acid protein assay kit to measure the total protein content (Beyotime Institute of Biotechnology, Inc.). Nuclear protein was extracted using a Nuclear Protein Extraction kit (cat. no. AR0106; Wuhan Boster Biological Technology, Ltd., Wuhan, China) according to the manufacturer's protocol. Following denaturation, equal amounts of protein ( $30 \mu \mathrm{g} / \mathrm{lane})$ were subjected to $10 \%$ SDS-PAGE and then transferred onto polyvinylidene difluoride membranes, which were washed in Tris-buffered saline with Tween-20 (TBST, 0.05\%) and blocked in 5\% skimmed milk (Sigma-Aldrich, Merck KGaA, Darmstadt, Germany) at $4^{\circ} \mathrm{C}$ overnight. Next, the membranes were incubated with rabbit

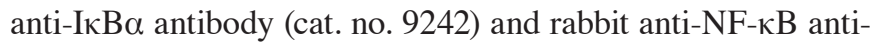
body (cat. no. 8242; both Cell Signaling Technology, Danvers, MA, USA) diluted at 1:1,000 in 5\% skimmed milk overnight at $4^{\circ} \mathrm{C}$ and subsequently washed three times for $15 \mathrm{~min}$ prior to being incubated with peroxidase-conjugated anti-mouse (cat. no. ZB-2305; OriGene Technologies, Inc., Beijing, China) or peroxidase-conjugated goat anti-rabbit immunoglobulin $\mathrm{G}$ (cat. no. ZB-2301; OriGene Technologies, Inc.) diluted at 1:500 at room temperature for $1 \mathrm{~h}$. The bound antibodies were visualised with an enhanced chemiluminescence (ECL) western blot detection system according to the manufacturer's protocol (GE Healthcare, Chalfont, UK).

Histopathological evaluation. Kidney specimens were collected and fixed in $10 \%$ formalin for $24 \mathrm{~h}$ at room temperature and then embedded in paraffin. Sections $4 \mu \mathrm{m}$ thick were cut from formalin-fixed tissues and stained with hematoxylin and eosin according to the manufacturer's protocol (cat. no. G1120; Beijing Solarbio Science \& Technology Co., Ltd., Beijing, China). Specimens were then examined under a light microscope at a magnification of $\mathrm{x} 200$.

Statistical analysis. Values are expressed as the mean \pm standard deviation. Statistical analysis was performed using a one-way ANOVA with a Dunnett's post hoc test as the multiple comparison method. $\mathrm{P}<0.05$ was considered to indicate a statistically significant difference. 


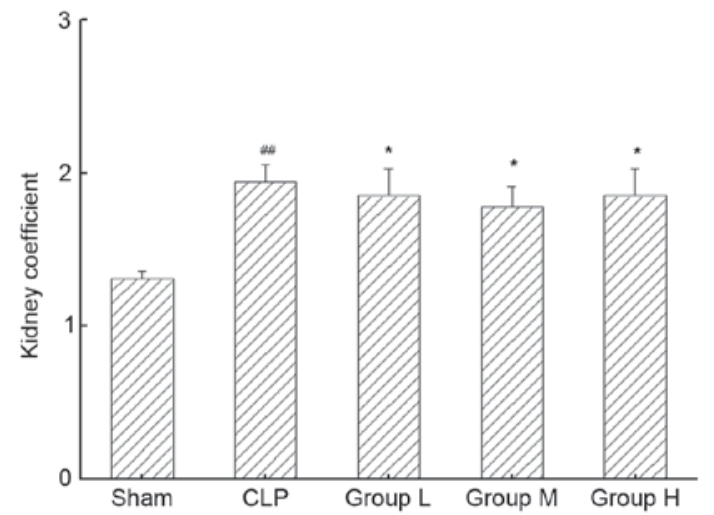

Figure 1. Kidney coefficients of mice in different treatment groups. Values are expressed as the mean \pm standard deviation $(\mathrm{n}=20)$. ${ }^{\# \#} \mathrm{P}<0.01$ vs. the sham group; ${ }^{*} \mathrm{P}<0.05$ vs. the CLP group. CLP, cecal ligation and puncture; $\mathrm{H}$, high-dose TNG (50 mg/kg); M, medium dose TNG (20 mg/kg); L, low-dose TNG (5 mg/kg); TNG, tenuigenin.

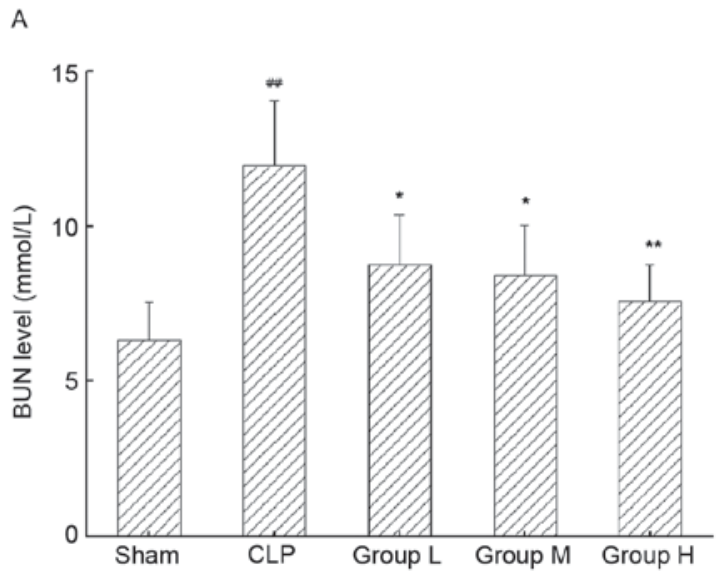

B

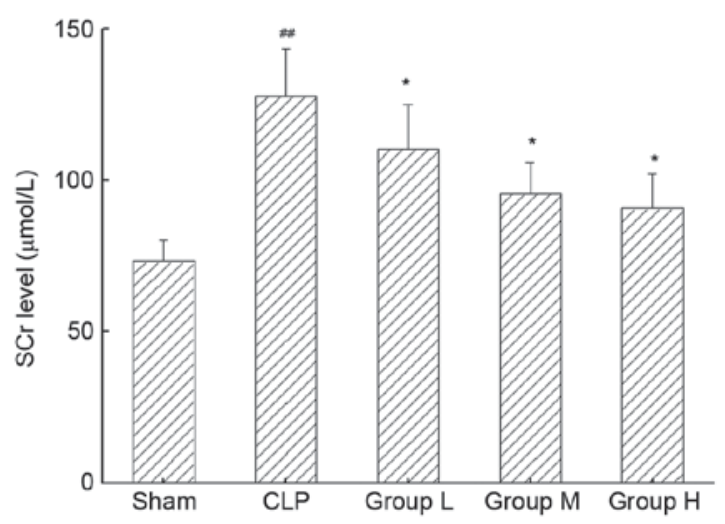

Figure 2. Effect of TNG on renal function of mice. (A) BUN and (B) SCr levels in different treatment groups. Values are expressed as the mean \pm standard deviation $(\mathrm{n}=20)$. ${ }^{\# \#} \mathrm{P}<0.01$ vs. the sham group; ${ }^{*} \mathrm{P}<0.05,{ }^{* *} \mathrm{P}<0.01$ vs. the CLP group. CLP, cecal ligation and puncture; $\mathrm{H}$, high-dose TNG $(50 \mathrm{mg} / \mathrm{kg})$; $\mathrm{M}$, medium dose TNG (20 mg/kg); L, low-dose TNG (5 mg/kg); TNG, tenuigenin; BUN, blood urea nitrogen; $\mathrm{SCr}$, serum creatinine.

\section{Results}

TNG treatment improves the kidney coefficient in mice with sepsis-induced AKI. Fig. 1 demonstrated the effects of TNG
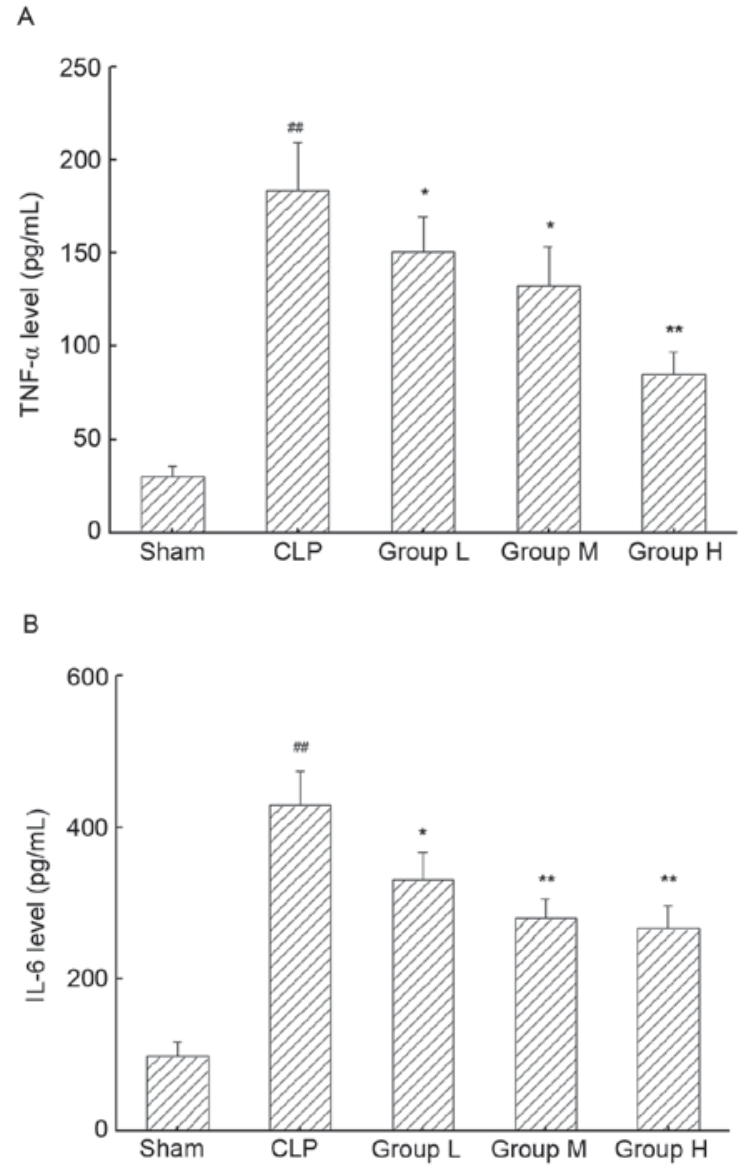

Figure 3. Effect of TNG on inflammatory cytokine production. Levels of (A) TNF- $\alpha$ and (B) IL- 6 in the kidneys were determined by ELISA. Values are expressed as the mean \pm standard deviation $(n=20)$. ${ }^{\# \#} \mathrm{P}<0.01$ vs. the sham group; ${ }^{*} \mathrm{P}<0.05,{ }^{* *} \mathrm{P}<0.01$ vs. the CLP group. TNF, tumor necrosis factor; IL, interleukin; CLP, cecal ligation and puncture; $\mathrm{H}$, high-dose TNG (50 mg/kg); M, medium dose TNG (20 mg/kg); L, low-dose TNG (5 mg/kg); TNG, tenuigenin.

treatment on the kidney coefficient in the different experimental groups. According to the results, the kidney coefficient in the CLP group was significantly higher than that in the sham group $(\mathrm{P}<0.01)$. TNG treatment caused marked reductions in the kidney coefficient in the L group compared with that in the CLP group $(\mathrm{P}<0.05)$ and this tendency was also present in the other two TNG treatment groups $(\mathrm{P}<0.05)$.

TNG improves kidney function parameters in mice with sepsis-induced AKI. The BUN and SCr levels in mice with sepsis-induced AKI were assessed (Fig. 2A and B, respectively). Following CLP surgery, mice with AKI displayed significant elevations in the levels of BUN $(\mathrm{P}<0.01)$ and $\mathrm{SCr}$ $(\mathrm{P}<0.01)$ in renal tissues. Of note, the elevated levels of BUN and $\mathrm{SCr}$ were significantly lowered by the application of TNG $(\mathrm{P}<0.05$ for each).

TNG treatment ameliorates inflammatory response in the kidneys of mice following CLP. To analyze the inflammatory response in the kidneys of mice, the levels of TNF- $\alpha$ and IL-6 in kidneys were assessed by ELISA (Fig. 3A and B, respectively). Compared with the sham group, TNF- $\alpha$ and IL-6 levels were significantly increased in the CLP group $(\mathrm{P}<0.01$ for 


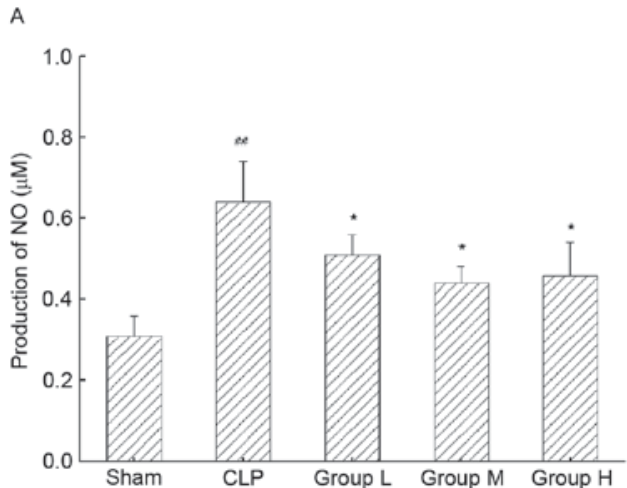

B

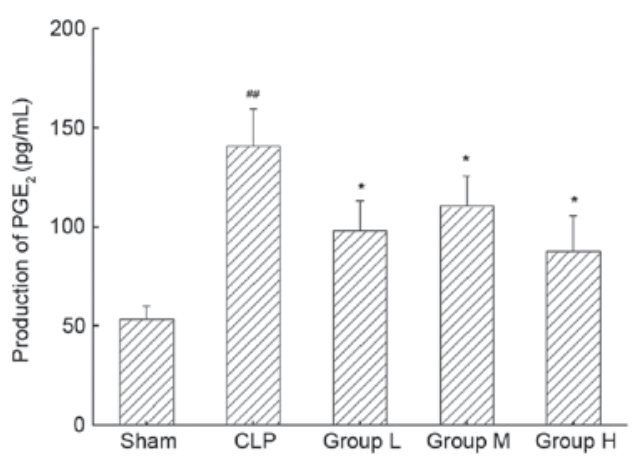

Figure 4. TNG inhibits the production of $\mathrm{NO}$ and $\mathrm{PGE}_{2}$ in kidney tissue induced by acute kidney injury. (A) Production of NO and (B) concentration of $\mathrm{PGE}_{2}$ in the kidneys of animals in different groups. Values are expressed as the mean \pm standard deviation $(\mathrm{n}=20)$. ${ }^{\# \#} \mathrm{P}<0.01$ vs. the sham group; ${ }^{*} \mathrm{P}<0.05$ vs. the CLP group. CLP, cecal ligation and puncture; $\mathrm{H}$, high-dose TNG (50 mg/kg); M, medium dose TNG (20 mg/kg); L, low-dose TNG (5 mg/kg); TNG, tenuigenin; NO, nitric oxide, PGE, prostaglandin E.

each), which was significantly inhibited by TNG treatment in a dose-dependent manner $(\mathrm{P}<0.05$ or $\mathrm{P}<0.01)$.

$T N G$ decreases the production of $N O$ and $P G E_{2}$ in mice with sepsis-induced AKI. To further demonstrate the anti-inflammatory effect of TNG, the production of inflammatory mediators and proteins was detected. As demonstrated in Fig. 4, sepsis resulted in a significant increase in NO production in kidney tissue compared with that in the sham group $(\mathrm{P}<0.01)$, whereas TNG significantly inhibited sepsis-induced production of NO ( $\mathrm{P}<0.05$; Fig. 4A). Furthermore, TNG significantly inhibited sepsis-induced production of $\mathrm{PGE}_{2}(\mathrm{P}<0.05$; Fig. 4B).

TNG attenuates histological changes in the kidneys of mice after CLP. Histological analysis of hematoxylin and eosin-stained kidney samples demonstrated a marked thickening of the glomerular basement membrane and an obvious expansion of mesangium in the CLP group compared with the sham group (Fig. 5A and B). Of note, these histopathological alterations in the kidney were improved in the TNG-treatment groups (Fig. 5C-E).

TNG inhibits the NF- $\kappa B$ signaling pathway in the kidneys of mice induced by $A K I$. To further evaluate the mechanisms underlying the anti-inflammatory effect of TNG, the level of nuclear $\mathrm{NF}-\kappa \mathrm{B}$ and that of its inhibitor $\mathrm{I} \kappa \mathrm{B} \alpha$ was assessed (Fig. 6). Western blot analysis demonstrated that the levels of
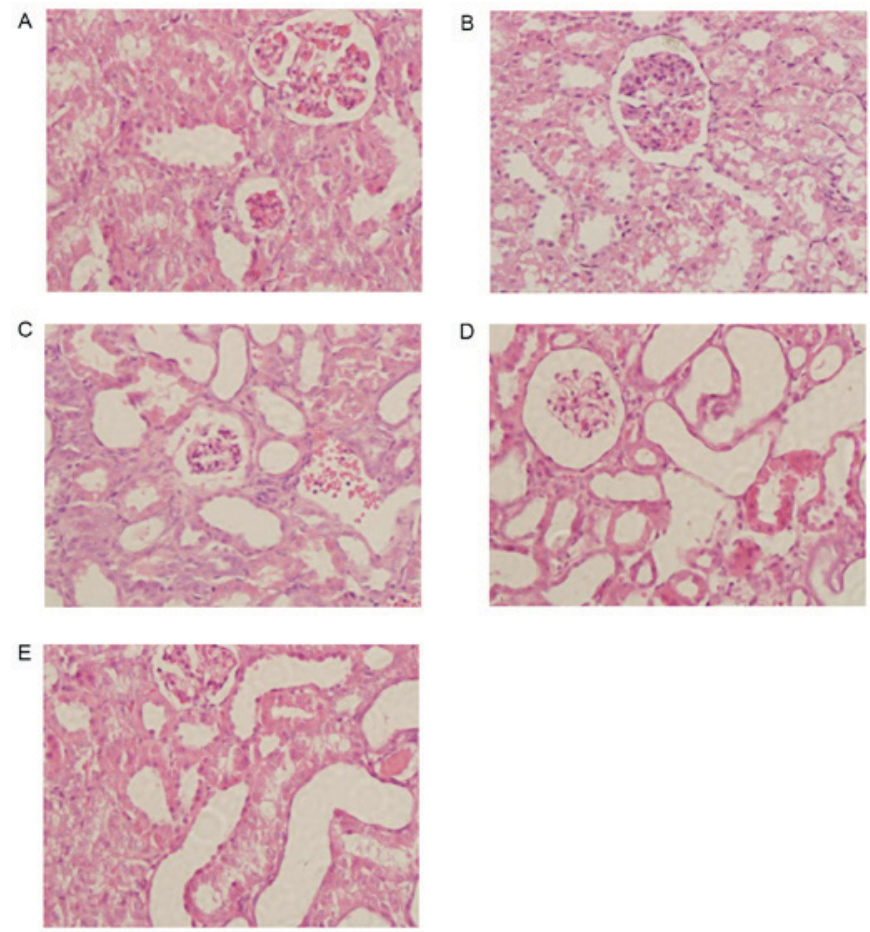

Figure 5. Histopathological changes in the kidneys of mice in different treatment groups (magnification, x20; hematoxylin and eosin staining). (A) Sham group; (B) cecal ligation and puncture group; (C) low-dose TNG (5 mg/kg) group; (D) medium dose TNG (20 mg/kg) group; (E) high-dose TNG $(50 \mathrm{mg} / \mathrm{kg})$ group. Arrows indicate histopathological alterations. The results shown are representative of at least three independent experiments. TNG, tenuigenin.

$\mathrm{NF}-\kappa \mathrm{B}$ in the nuclei of kidney cells from mice in the CLP group were significantly increased compared with those in the sham group $(\mathrm{P}<0.05)$, while the expression of $\mathrm{I} \kappa \mathrm{B} \alpha$ was decreased $(\mathrm{P}<0.01)$. While middle and high dose of TNG treatment significantly increased $\mathrm{I} \kappa \mathrm{B} \alpha$ expression compared to that in the CLP group. I $\mathrm{B} \alpha$ expression in the $\mathrm{H}$ group was slightly decreased compared with that of the $\mathrm{M}$ group, however, the difference was not statistically significant. Following treatment with different doses of TNG, although the NF- $\mathrm{NB}$ levels in the three TNG treatment groups remained higher than that in the sham group $(\mathrm{P}<0.01)$, but were significantly decreased when compared with the CLP group $(\mathrm{P}<0.01)$.

\section{Discussion}

Inflammation is an essential component of the innate immune response that protects against tissue damage. However, when unbalanced, inflammation causes tissue damage (17). AKI is thought to be a result of uncontrolled renal inflammation and has a high mortality rate. Traditional Chinese medicines have been used for the prevention and treatment of various diseases in China for several thousands of years. TNG is an active component of a traditional Chinese medicinal herb that has been reported to have anti-oxidant and anti-inflammatory properties $(13,14)$. The present study examined whether the protective effect of TNG on AKI induced by CLP is due to its anti-inflammatory properties.

The present study demonstrated that treatment with TNG in mice with sepsis-induced AKI alleviated the pathological 
A

$\mathrm{I \kappa B} \alpha$

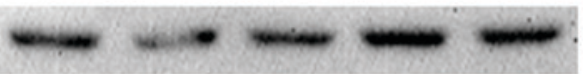

$\beta$-actin

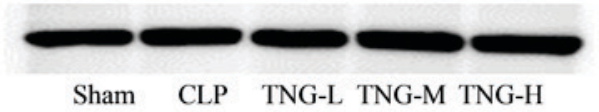

B

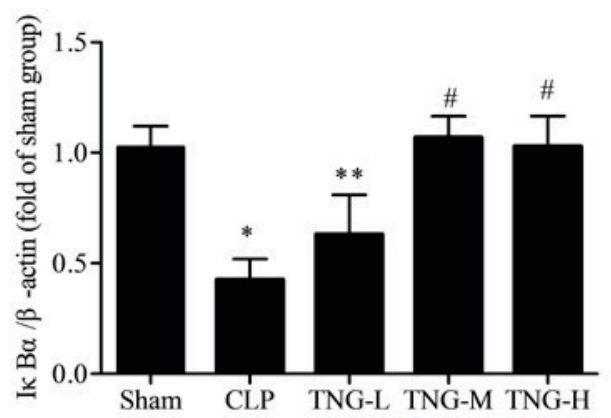

Neucleus NF-кB

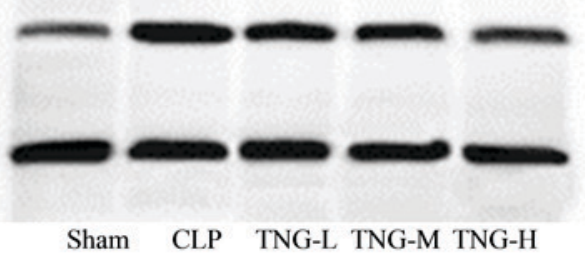

Histone

Sham

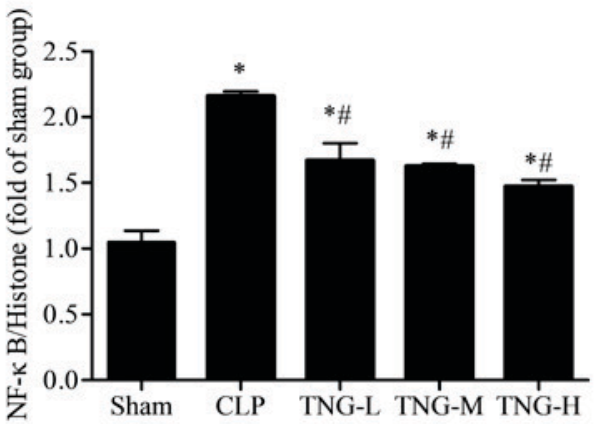

Figure 6. TNG inhibits the activation of NF- $\kappa$ B induced by acute kidney injury. (A) The expression of proteins associated with the NF- $\kappa \mathrm{B}$ signaling pathway was detected by western blot analysis. $\beta$-actin and Histone $\mathrm{H}$ were used as loading controls. (B) Quantified expression of proteins associated with the NF- $\mathrm{B}$ signaling pathway. Data are expressed as the mean \pm standard deviation of three independent experiments. CLP, cecal ligation and puncture; $\mathrm{H}$, high-dose TNG (50 mg/kg); M, medium dose TNG (20 mg/kg); L, low-dose TNG (5 mg/kg); TNG, tenuigenin; NF, nuclear factor; I $\kappa B$, inhibitor of NF- $\mathrm{BB}$. * $<<0.01 \mathrm{vs.} \mathrm{sham}$ group; ${ }^{* *} \mathrm{P}<0.05$ vs. sham group; ${ }^{*} \mathrm{P}<0.01$ vs. CLP group.

damage of kidney tissue, attenuated renal dysfunction and inhibited the release of inflammatory cytokines and mediators, indicating a profound impact on the severity of AKI. To shed light on the underlying mechanisms, the present study focused on the NF- $\kappa \mathrm{B}$ pathway. The results demonstrated that TNG significantly inhibited the activation of $\mathrm{NF}-\kappa \mathrm{B}$ the in kidneys of mice challenged by CLP. These results indicated that TNG exerts its anti-inflammatory effects at least in part through inhibition of the activation of the $\mathrm{NF}-\kappa \mathrm{B}$ signaling pathway.

The present study established a mouse model of sepsis-induced AKI, which is one of the classical methods for experimental studies on sepsis and also the animal model, which is the most representative of the pathophysiological processes of sepsis. The kidney is the most sensitive organ in sepsis. A progressive decline in the glomerular filtration rate, reflecting $\mathrm{SCr}$ and BUN levels, is the most common characteristic in the development of AKI, which causes proteinuria, leading to histological damage in the kidney. Following CLP surgery, mice with AKI exhibited significant increases in $\mathrm{SCr}$ and BUN levels and pathological abnormalities were observed, representing a decline in renal function. However, TNG treatment positively affected these parameters of renal function and effectively restrained the pathological changes, indicating a renoprotective effect of TNG in sepsis-induced AKI.

There is a strong association between inflammatory cytokine levels and the development of sepsis-induced AKI $(18,19)$. Therefore, the upregulation of inflammatory cytokines, including TNF- $\alpha$ and IL- 6 is considered a reliable marker of sepsis. The experimental data of the presents study suggested that the production of TNF- $\alpha$ and IL- 6 was significantly induced by AKI, and was suppressed by treatment with TNG. Based on the above outcome, it was further demonstrated that TNG had a protective effect on mice with sepsis-induced AKI.

NO is derived from the oxidation of L-arginine, which is catalyzed by nitric oxide synthase (NOS). In sepsis, the expression of inducible (i)NOS induced by inflammatory mediators and cytokines is significantly increased in immunocytes, such as neutrophils and macrophages (20). In the present study, the production of NO was significantly induced by AKI, which was suppressed by treatment with TNG. $\mathrm{PGE}_{2}$ is an important inflammatory mediator and has a significant role in the inflammatory response $(21,22)$. In the present study, the production of PGE2 was significantly induced by AKI, which was suppressed by treatment with TNG.

The NF-kB pathway involves an important family of transcription factors that control the expression of cytokines, cell adhesion molecules, growth factors and also apoptosis, cell proliferation, differentiation and survival $(23,24)$. Studies have demonstrated the activation of $\mathrm{NF}-\kappa \mathrm{B}$ in the kidneys during AKI (25-27). The principal pro-inflammatory mediators in the pathophysiology of sepsis are TNF- $\alpha$ and IL- $1 \beta$, which activate $\mathrm{NF}-\kappa \mathrm{B}$ by triggering a signaling pathway that leads to the phosphorylation and consequent degradation of the inhibitor of NF- $\kappa \mathrm{B} \alpha(\mathrm{I} \kappa \mathrm{B} \alpha)(28,29)$. The degradation of I $\mathrm{B} \mathrm{B} \alpha$ generates a nuclear localization signal for the $\mathrm{NF}-\kappa \mathrm{B}$ protein, which then migrates into the nucleus and stimulates the transcription of specific genes. The overproduction of pro-inflammatory mediators enhances adhesion molecules and also leads to deleterious effects associated with multiple organ failure and shock (30). iNOS and cyclooxygenase (COX)-2 are two important proteins downstream of the $\mathrm{NF}-\kappa \mathrm{B}$ signaling pathway and $\mathrm{NF}-\kappa \mathrm{B}$ specifically binds to the promoter region of iNOS and COX-2, which promotes their transcription and ultimately facilitates the production of $\mathrm{NO}$ and $\mathrm{PGE}_{2}$. The present study demonstrated that following CLP, accumulation of NF- $\kappa \mathrm{B}$ was increased, accompanied with downregulation of the expression of I $\mathrm{B} \alpha$. As expected, following treatment with TNG, the $\mathrm{NF}-\kappa \mathrm{B}$ signaling pathway was inhibited.

In conclusion, the results of the present study indicated that TNG significantly ameliorates kidney tissue damage, improves 
renal function, and inhibits the release of inflammatory cytokines and mediators, suggesting that TNG has a protective effect on AKI caused by sepsis. This renoprotection of TNG may be due to its anti-inflammatory effects. The present study also provided evidence that the mechanism of action may involve the inhibition of the NF- $\kappa \mathrm{B}$ signaling pathway. These results indicated that TNG is a promising candidate for a novel adjuvant therapeutic strategy for sepsis-induced AKI and that the NF- $\mathrm{NB}$ signaling pathway may be a potential target.

\section{References}

1. Lauer S, Fischer LG, Van Aken HK, Nofer JR and Freise H: Gadolinium chloride modulates bradykinin-induced pulmonary vasoconstriction and hypoxic pulmonary vasoconstriction during polymicrobial abdominal sepsis in rats. Exp Lung Res 41: 270-282, 2015.

2. Bagshaw SM, Uchino S, Bellomo R, Morimatsu H, Morgera S, Schetz M, Tan I, Bouman C, Macedo E, Gibney N, et al: Septic acute kidney injury in critically ill patients: Clinical characteristics and outcomes. Clin J Am Soc Nephrol 2: 431-439, 2007.

3. Mårtensson $\mathrm{J}$ and Bellomo R: Sepsis-induced acute kidney injury. Crit Care Clin 31: 649-660, 2015.

4. Zarjou A and Agarwal A: Sepsis and acute kidney injury. J Am Soc Nephrol 22: 999-1006, 2011.

5. Singbartl K and Kellum JA: AKI in the ICU: definition, epidemiology, risk stratification, and outcomes. Kidney Int 81: 819-825 2012.

6. Schrier RW and Wang W: Acute renal failure and sepsis. N Engl J Med 351: 159-169, 2004.

7. Kellum JA, Kong L, Fink MP, Weissfeld LA, Yealy DM, Pinsky MR, Fine J, Krichevsky A, Delude RL and Angus DC; GenIMS Investigators: Understanding the inflammatory cytokine response in pneumonia and sepsis: Results of the Genetic and Inflammatory Markers of Sepsis (GenIMS) Study. Arch Intern Med 167: 1655-1663, 2007.

8. Tsujimoto H, Ono S, Efron PA, Scumpia PO, Moldawer LL and Mochizuki H: Role of Toll-like receptors in the development of sepsis. Shock 29: 315-321, 2008.

9. Hotchkiss RS and Karl IE: The pathophysiology and treatment of sepsis. N Engl J Med 348: 138-150, 2003.

10. Liu SF and Malik AB: NF- $\kappa$ B activation as a pathological mechanism of septic shock and inflammation. Am J Physiol Lung Cell Mol Physiol 290: L622-L645, 2006.

11. Christaki E, Anyfanti P and Opal SM: Immunomodulatory therapy for sepsis: an update. Expert Rev Anti Infect Ther 9: 1013-1033, 2011.

12. Park CH, Choi SH, Koo JW, Seo JH, Kim HS, Jeong SJ and Suh YH: Novel cognitive improving and neuroprotective activities of Polygala tenuifolia Willdenow extract, BT-11. J Neurosci Res 70: 484-492, 2002.

13. Yuan HL, Li B, Xu J, Wang Y, He Y, Zheng Y and Wang XM: Tenuigenin protects dopaminergic neurons from inflammation mediated damage induced by the lipopolysaccharide. CNS Neurosci Ther 18: 584-590, 2012.
14. Zhang H, Han T, Zhang L, Yu CH, Wan DG, Rahman K, Qin LP and Peng C: Effects of tenuifolin extracted from radix polygalae on learning and memory: A behavioral and biochemical study on aged and amnesic mice. Phytomedicine 15: 587-594, 2008.

15. National Research Council (US) Committee for the Update of the Guide for the Care and Use of Laboratory Animals: Guide for the Care and Use of Laboratory Animals. 8th edition. National Academies Press (US), Washington, DC, 2011.

16. Rittirsch D, Huber-Lang MS, Flierl MA and Ward PA: Immunodesign of experimental sepsis by cecal ligation and puncture. Nat Protoc 4: 31-36, 2009.

17. Serhan $\mathrm{CN}$, Chiang $\mathrm{N}$ and Van Dyke TE: Resolving inflammation: Dual anti-inflammatory and pro-resolution lipid mediators. Nat Rev Immunol 8: 349-361, 2008.

18. Murugan R, Karajala-Subramanyam V, Lee M, Yende S, Kong L, Carter M, Angus DC and Kellum JA: Acute kidney injury in non-severe pneumonia is associated with an increased immune response and lower survival. Kidney Int 77: 527-535, 2010.

19. Payen D, Lukaszewicz AC, Legrand M, Gayat E, Faivre V, Megarbane B, Azoulay E, Fieux F, Charron D, et al: A multicentre study of acute kidney injury in severe sepsis and septic shock: Association with inflammatory phenotype and HLA genotype. PLoS One 7: e35838, 2012.

20. Heemskerk S, Masereeuw R, Russel FG and Pickkers P: Selective iNOS inhibition for the treatment of sepsis-induced acute kidney injury. Nat Rev Nephrol 5: 629-640, 2009.

21. Ricciotti E and FitzGerald GA: Prostaglandins and Inflammation. Arterioscler Thromb Vasc Biol 31: 986-1000, 2011.

22. Correa M, Machado J Jr, Carneiro CR, Pesquero JB, Bader M, Travassos LR, Chammas R and Jasiulionis MG: Transient inflammatory response induced by apoptotic cells is an important mediator of melanoma cell engraftment and growth. Int J Cancer 114: 356-63, 2005.

23. Perkins ND: Integrating cell-signalling pathways with NF-кB and IKK function. Nat Rev Mol Cell Biol 8: 49-62, 2007.

24. Hayden MS and Ghosh S: Shared principles in NF- $\kappa B$ signaling. Cell 132: 344-362, 2008.

25. Basak S and Hoffmann A: Crosstalk via the NF-kappaB signaling system. Cytokine Growth Factor Rev 19: 187-197, 2008.

26. Benedetti G, Fokkelman M, Yan K, Fredriksson L, Herpers B, Meerman J, van de Water B and de Graauw M: The Nuclear Factor $\kappa \mathrm{B}$ Family Member RelB Facilitates Apoptosis of Renal Epithelial Cells Caused by Cisplatin/Tumor Necrosis Factor $\alpha$ Synergy by Suppressing an Epithelial to Mesenchymal Transition-Like Phenotypic Switch. Mol Pharmacol 84: 128-138, 2013.

27. Al-Lamki RS, Lu W, Finlay S, Twohig JP, Wang EC, Tolkovsky AM and Bradley JR: DR3 signaling protects against cisplatin nephrotoxicity mediated by tumor necrosis factor. Am J Pathol 180: 1454-1464, 2012.

28. Nozaki Y, Nikolic-Paterson DJ, Yagita H, Akiba H, Holdsworth SR and Kitching AR: Tim-1 promotes cisplatin nephrotoxicity. Am J Physiol Renal Physiol 301: F1098-F1104, 2011.

29. Chaffey N, Alberts B, Johnson A, Lewis J, Raff M, Roberts K and Walter P: Molecular biology of the cell. Ann Bot 91: 401, 2003.

30. Thijs A and Thijs LG: Pathogenesis of renal failure in sepsis. Kidney Int Suppl 66 (Suppl): S34-S37, 1998 